\title{
Effect of febuxostat on left ventricular diastolic function in patients with asymptomatic hyperuricemia: a sub analysis of the PRIZE Study
}

\author{
Kenya Kusunose ${ }^{1} \cdot$ Hisako Yoshida $^{2} \cdot$ Atsushi Tanaka $^{3} \cdot$ Hiroki Teragawa $^{4} \cdot$ Yuichi Akasaki $^{5} \cdot$ Yoshihiro Fukumoto $^{6}$. \\ Kazuo Eguchi ${ }^{7} \cdot$ Haruo Kamiya $^{8} \cdot$ Kazuomi Kario $^{9} \cdot$ Hirotsugu Yamada $^{10} \cdot$ Masataka Sata $^{1} \cdot$ Koichi Node $^{3} \cdot$ The PRIZE $^{3}$ \\ Study Investigators
}

Received: 27 August 2021 / Revised: 1 September 2021 / Accepted: 2 September 2021 / Published online: 17 October 2021

(c) The Author(s) 2021. This article is published with open access

\begin{abstract}
Hyperuricemia is related to an increased risk of cardiovascular events from a meta-analysis and antihyperuricemia agents may influence to cardiac function. We evaluated the effect of febuxostat on echocardiographic parameters of diastolic function in patients with asymptomatic hyperuricemia as a prespecified endpoint in the subanalysis of the PRIZE study. Patients in the PRIZE study were assigned randomly to either add-on febuxostat treatment group or control group with only appropriate lifestyle modification. Of the 514 patients in the overall study, 65 patients (31 in the febuxostat group and 34 in the control group) who had complete follow-up echocardiographic data of the ratio of peak early diastolic transmitral flow velocity (E) to peak early diastolic mitral annular velocity $\left(\mathrm{e}^{\prime}\right)$ at baseline and after 12 and 24 months were included. The primary endpoint was a comparison of the changes in the $\mathrm{E} / \mathrm{e}^{\prime}$ between the two groups from baseline to 24 months. Interestingly, $\mathrm{e}^{\prime}$ was slightly decreased in the control group compared with in the febuxostat group (treatment $p=0.068$, time, $p=0.337$, treatment $\times$ Time, $p=0.217$ ). As a result, there were significant increases in $\mathrm{E} / \mathrm{e}^{\prime}$ (treatment $p=0.045$, time, $p=0.177$, treatment $\times$ time, $p=0.137$ ) after 24 months in the control group compared with the febuxostat group. There was no significant difference in the serum levels of $\mathrm{N}$-terminal-pro brain natriuretic peptide and high-sensitive troponin I between the two groups during the study period. In conclusions, additional febuxostat treatment in patients with asymptomatic hyperuricemia for 24 months might have a potential of preventable effects on the impaired diastolic dysfunction.
\end{abstract}

Keywords febuxostat $\cdot$ hyperuricemia $\cdot$ echocardiography $\cdot$ diastolic function $\cdot$ NT-proBNP

\section{Introduction}

A list of authors and their affiliations appears below the

Acknowledgement Section.

Kenya Kusunose

kusunosek@tokushima-u.ac.jp

1 Department of Cardiovascular Medicine, Tokushima University Hospital, Tokushima, Japan

2 Department of Medical Statistics, Osaka City University Graduate School of Medicine, Osaka, Japan

3 Department of Cardiovascular Medicine, Saga University, Saga, Japan

4 Department of Cardiovascular Medicine, JR Hiroshima Hospital, Hiroshima, Japan

5 Department of Cardiovascular Medicine and Hypertension, Graduate School of Medical and Dental Sciences, Kagoshima University, Kagoshima, Japan
Hyperuricemia can be associated with cardiometabolic abnormalities and the association leads to the development of atherosclerosis and resultant cardiovascular disease

6 Division of Cardiovascular Medicine, Department of Medicine, Kurume University School of Medicine, Kurume, Japan

7 Department of General Internal Medicine, Saitama Red Cross Hospital, Saitama, Japan

8 Department of Cardiology, Japanese Red Cross Aichi Medical Center Nagoya Daiichi Hospital, Nagoya, Japan

9 Division of Cardiovascular Medicine, Department of Medicine, Jichi Medical University School of Medicine, Shimotsuke, Japan

10 Department of Community Medicine for Cardiology, Tokushima University Graduate School of Biomedical Sciences,

Tokushima, Japan 
[1-3]. Several studies investigated the association between hyperuricemia and echocardiographic variables [4-7]. Krishnan et al. showed the correlation between the left ventricular (LV) mass index and serum uric acid (SUA) level [8]. In addition, Lin et al. also showed the LV diastolic functional parameters were associated with gout [9]. The association between hyperuricemia and cardiac function is a key mechanism of hyperuricemia on cardiovascular diseases. Therefore, it is clinically required to evaluate the impact of antihyperuricemia agents on cardiac function from the randomized cohort study.

Febuxostat is a nonpurine selective inhibitor of xanthine oxidase for the treatment of hyperuricemia and gout. Febuxostat has greater potency for inhibition of xanthine oxidase activity and more urate-lowering efficacy than allopurinol. Accordingly, febuxostat may have superior antioxidative and antiatherogenic effects to allopurinol $[10,11]$. According to the link between hyperuricemia and cardiac function, we hypothesized that the febuxostat can influence the LV diastolic function in patients with hyperuricemia. The PRIZE (program of vascular evaluation under uric acid control by xanthine oxidase inhibitor, febuxostat: multicenter, randomized controlled) study (University Hospital Medical Information Network Clinical Trial Registry UMIN000012911) was a prospective multicenter study conducted in Japanese patients with asymptomatic hyperuricemia to evaluate the inhibitory effect of febuxostat on the progression of atherosclerosis based on carotid-artery intima-media thickness assessed by ultrasonography over a 2-year follow-up period [12]. To elucidate the effect of febuxostat on echocardiographic parameters, we investigated the effect of the febuxostat on 2-dimensional and Doppler echocardiographic parameters and biomarkers from baseline to 24 months as a prespecified endpoint in the subanalysis of the PRIZE study (University Hospital Medical Information Network Clinical Trial Registry UMIN000041322).

\section{Study design}

Details of the PRIZE study design and inclusion and exclusion criteria for the study have been published elsewhere [13]. In brief, the study was a multicenter, randomized, prospective, open-label, blinded-endpoint trial accomplished by 48 sites throughout Japan. A total of 514 adults (aged $\geq 20$ years) who had hyperuricemia with SUA $>7.0 \mathrm{mg} / \mathrm{dL}$ enrolled in this study between May 2014 and August 2018. Key exclusion criteria were the administration of any SUA-lowering agents within the 8-week period prior to the assessment of eligibility, the presence of gouty tophus, or symptoms of gout arthritis within one year before assessment of eligibility. The patients were randomly assigned using a 1:1 ratio to either add-on febuxostat treatment (febuxostat group, $n=257$ ) or an appropriate lifestyle modification for hyperuricemia, such as healthy diet and exercise therapy without febuxostat (control group, $n$ $=257$ ). The original primary endpoint of the PRIZE study was the change in mean common carotid IMT 24 months after treatment randomization. We prespecified the echocardiographic endpoint in the subanalysis of the PRIZE study. Echocardiography was performed as an ad hoc examination at baseline and 12 and 24 months after treatment randomization. The ethical committees of each participating institution approved the study protocol, with written informed consent for participation in the study being obtained from all subjects.

\section{Study population}

The echocardiographic examination was performed in 59 patients in the febuxostat group and in 63 patients in the control group at the baseline. We excluded the patients with the lack of echocardiographic data at both 12 and 24 months to analyze the changes of echocardiographic parameters during follow-up. After exclusions, 31 patients in the febuxostat group and in 34 patients in the control group were included for the final analysis (Fig. 1).

\section{Echocardiographic assessment}

Echocardiography was performed by the commercially available ultrasound machine and various hemodynamic parameters were measured at each institution. The recordings and measurements were according to the guidelines issued by American Society of Echocardiography [14]. Left ventricular ejection fraction (LVEF) and left atrial volume were measured and calculated from the apical two- and four-chamber view using bi-plane disk methods. LV mass by M-mode echocardiography was derived using the American Society of Echocardiography's guideline formula. Transmitral flow velocity was recorded from the apical long-axis or four-chamber view. The peak early diastolic (E) and the peak atrial systolic (A) velocities, and deceleration time of early TMF velocity were measured. The mitral annular motion velocity pattern was recorded from the apical four-chamber view with a sample volume placed at the lateral or septal side of the mitral annulus using pulsed tissue Doppler echocardiography. Early diastolic $\left(\mathrm{e}^{\prime}\right)$ peak velocities were measured and the ratio of $\mathrm{E}$ to $\mathrm{e}^{\prime}\left(\mathrm{E} / \mathrm{e}^{\prime}\right)$ was calculated using averaged values. This index was used to be a marker of LV filling pressure.

\section{Laboratory examination}

Blood samples were collected at baseline and after 12 and 24 months. The serum levels of $\mathrm{N}$-terminal pro-brain natriuretic peptide (NT-proBNP) and high-sensitive troponin I were measured in a centralized laboratory (SRL Co. Tokyo, Japan). 
Fig. 1 Patient selection

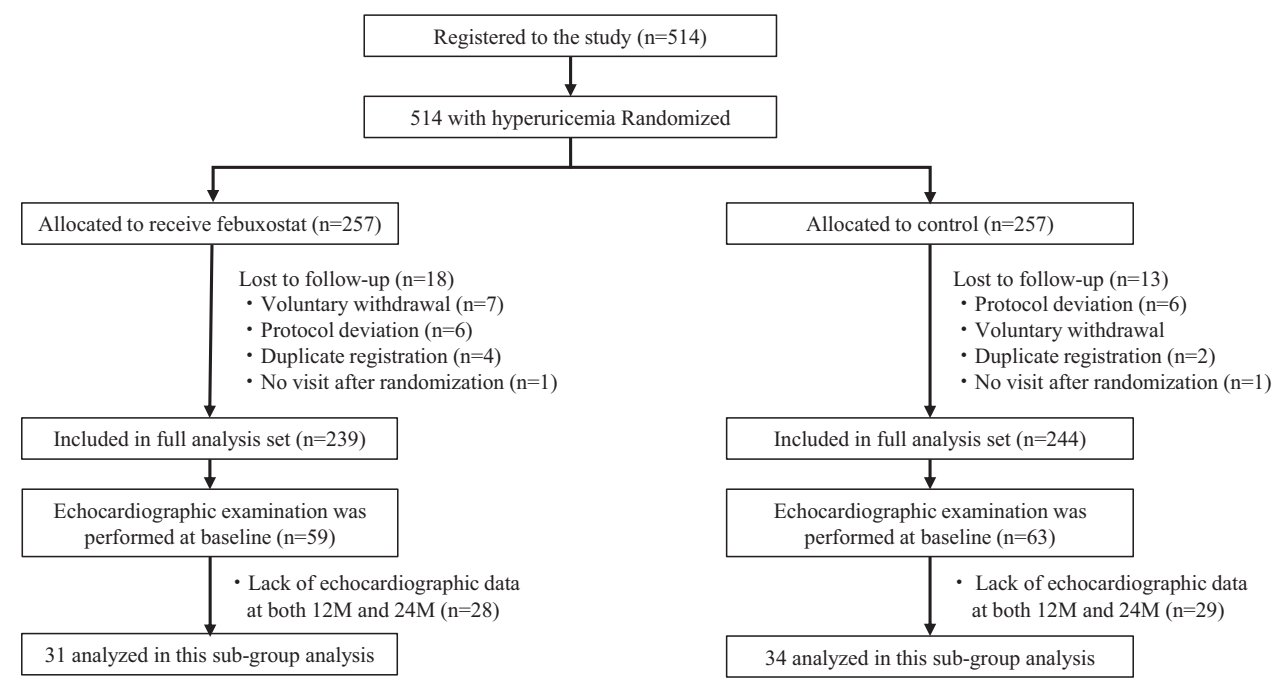

\section{Statistical analysis}

For the baseline variables, the summary statistics were expressed as frequencies and proportions for categorical data and mean (SD) or median (interquartile range) for continuous variables. Mean percentage changes in the echocardiographic variables and laboratory parameters from baseline to 12 and 24 months and its $95 \%$ confidence interval (CI), estimated with the repeated measures regression model using the mixed-effect model. To assess change from the baseline in the model with the logarithmic the echocardiographic variables, the baseline logarithmic variable was further adjusted in the model. Comparisons of changes in the other efficacy endpoints between the treatment groups were performed by examining the interaction between the treatment groups and follow-up time, which statistical tests for differences in rates of change between the regression slopes of the weight over time between the febuxostat group and control group. All P values were twosided with a level of significance of 0.05 , and there were adjustments for baseline data. We were unable to adjust for the other variables in this analysis due to the limited number of patients. All statistical analyses were performed using R 4.0.1. (R Foundation for Statistical Computing, Vienna, Austria.)

\section{Results}

\section{Baseline clinical characteristics}

Comparisons of baseline clinical characteristics of the febuxostat group and control group were shown in Table 1 . In the febuxostat group, $25.8 \%$ of the patients received 10 $\mathrm{mg}, 22.6 \%$ received $20 \mathrm{mg}$, and $48.4 \%$ received $40 \mathrm{mg}$ daily as the final dose of febuxostat. While around $90 \%$ of the subjects had hypertension in the two groups, the blood pressure was well controlled in both groups. Dyslipidemia also existed in around $60 \%$ of the patients in each group; however, the average serum total cholesterol level was within the normal range. There was no significant difference in any examined variable between the two groups at baseline except the estimated glomerular filtration rate which was slightly higher in the control group than in the febuxostat group $(p=0.020)$. The uric acid level was significantly lower in the control group $(p=0.031)$. Table 2 showed the baseline echocardiographic variables in both groups. At baseline, averaged $\mathrm{e}^{\prime}$ in febuxostat group was lower and E/e' in the febuxostat group was higher than in the control group. There was no significant difference in the other variables between the two groups. Around 70\% patients had normal or concentric remodeling LV geometry.

\section{Laboratory data}

Figure 2 showed the changes in laboratory data, including uric acid, NT-pro BNP, and high-sensitive troponin I. There were significant differences in the SUA levels between the treatment groups at 6,12 , and 24 months, and the final SUA levels were 4.46 and $7.07 \mathrm{mg} / \mathrm{dL}$ in the febuxostat and control groups, respectively (treatment, time and treatment $\times$ time, all $p<0.001)$. There were no significant differences in serum level of NT-pro BNP (treatment $p=$ 0.1143 ) and high-sensitive troponin I (treatment $p=0.547$ ) in both groups throughout this study.

\section{Echocardiographic parameters}

Comparisons of echocardiographic parameters at baseline and after 12 and 24 months of treatment in the febuxostat group and control group are shown in Figs. 3 and 4. There were no significant differences of $\mathrm{LV}$ ejection fraction (treatment $p=0.422$, time, $p=0.283$, treatment $\times$ Time, $p$ $=0.469$; Fig. 3) and LV mass index (treatment $p=0.713$, 
Table 1 Clinical characteristics at baseline

\begin{tabular}{|c|c|c|c|c|}
\hline & ALL & Control & Febuxostat & $P$ value \\
\hline Number & 65 & 34 & 31 & \\
\hline \multicolumn{5}{|l|}{ Clinical background } \\
\hline Age, year & $71[63,77]$ & $71[63,77]$ & $71[63,77]$ & 1.000 \\
\hline Male, $\%$ & $80(52)$ & $88(30)$ & $71(22)$ & 0.082 \\
\hline Body mass index & $25.1[22.2,26.9]$ & $25.6[22.9,27.4]$ & $24.7[22.1,26.4]$ & 0.482 \\
\hline Systolic BP, mmHg & $128[118,133]$ & $128[120,132]$ & $126[115,133]$ & 0.818 \\
\hline Diastolic BP, mmHg & $72[66,80]$ & $77[68,80]$ & $72[65,79]$ & 0.252 \\
\hline Heart rate, bpm & $62[58,72]$ & $63[56,71]$ & $62[59,72]$ & 0.555 \\
\hline \multicolumn{5}{|l|}{ Clinical history } \\
\hline Smoker, $\%,(n)$ & $55.4(36)$ & $64.7(22)$ & $45.2(14)$ & 0.113 \\
\hline Hypertension, $\%,(n)$ & $90.8(59)$ & $88.2(30)$ & $93.5(29)$ & 0.460 \\
\hline Diabetes mellitus, $\%,(n)$ & $41.5(27)$ & $38.2(13)$ & $45.2(14)$ & 0.571 \\
\hline Dyslipidemia, $\%,(n)$ & $63.1(41)$ & $55.9(19)$ & $71.0(22)$ & 0.208 \\
\hline Myocardial infarction, $\%,(n)$ & $13.8(9)$ & $8.8(3)$ & $19.4(6)$ & 0.220 \\
\hline PCI, $\%,(n)$ & $24.6(16)$ & $17.6(6)$ & $32.3(10)$ & 0.172 \\
\hline $\mathrm{CABG}, \%,(n)$ & $6.2(4)$ & $5.9(2)$ & $6.5(2)$ & 0.924 \\
\hline Stroke, $\%,(n)$ & $4.6(3)$ & $5.9(2)$ & $3.2(1)$ & 0.610 \\
\hline Heart failure, $\%,(n)$ & $16.9(11)$ & $8.8(3)$ & $25.8(8)$ & 0.068 \\
\hline \multicolumn{5}{|l|}{ Medications } \\
\hline $\mathrm{ARB} \%,(n)$ & $61.5(40)$ & $58.8(20)$ & $64.5(20)$ & 0.638 \\
\hline ACEI $\%,(n)$ & $12.8(8)$ & $11.8(4)$ & $12.9(4)$ & 0.889 \\
\hline Beta blocker $\%,(n)$ & $44.6(29)$ & $45.1(15)$ & $45.2(14)$ & 0.933 \\
\hline Diuretic $\%,(n)$ & $33.8(22)$ & $32.4(11)$ & $35.5(11)$ & 0.790 \\
\hline Statin $\%,(n)$ & $47.7(31)$ & $44.1(15)$ & $51.6(16)$ & 0.610 \\
\hline Anti-platelet $\%,(n)$ & $47.7(31)$ & $50.0(17)$ & $45.2(14)$ & 0.696 \\
\hline Aspirin \%, (n) & $41.5(27)$ & $38.2(13)$ & $45.2(14)$ & 0.571 \\
\hline \multicolumn{5}{|l|}{ Laboratory Data } \\
\hline Total cholesterol, mmol/L & $4.56[4.01,5.25]$ & $4.55[4.16,5.25]$ & $4.58[3.98,5.31]$ & 0.968 \\
\hline $\mathrm{eGFR}, \mathrm{mL} / \mathrm{min} / 1.73 \mathrm{~m}^{2}$ & $57.5[47.0,66.8]$ & $60.1[53.2,68.9]$ & $48.4[42.5,59.7]$ & 0.020 \\
\hline Uric acid, mg/dL & $7.65[7.10,8.28]$ & $7.50[7.10,7.90]$ & $8.10[7.35,8.70]$ & 0.031 \\
\hline NT-proBNP, pg/mL & $99.5[33.3,314.1]$ & $98.7[33.3,222.4]$ & $100.4[37.3,361.4]$ & 0.624 \\
\hline Troponin I, pg/mL & $5.15[3.20,9.70]$ & $5.10[3.30,8.00]$ & $5.30[3.15,10.55]$ & 0.867 \\
\hline
\end{tabular}

$B P$ blood pressure, $P C I$ percutaneous coronary intervention, $A C E i$ angiotensin-converting-enzyme inhibitor, $A R B$ angiotensin II receptor blocker, $e G F R$ estimate glomerular filtration rate, $N T$-proBNP N-terminal-pro brain natriuretic peptide.

Data are presented as the number of patients (percentage), mean \pm SD or median (interquartile range). time, $p=0.532$, treatment $\times$ Time, $p=0.778$; Fig. 3) at baseline and after 12 and 24 months in both groups. In the Doppler parameters, there were no significant difference of E wave (treatment $p=0.758$, time, $p=0.325$, treatment $\times$ Time, $p=0.486$; Fig. 4) at baseline and after 12 and 24 months in both groups. Interestingly, e' was slightly decreased in the control group compared with in the febuxostat group (treatment $p=0.068$, time, $p=0.337$, treatment $\times$ Time, $p=0.217$; Fig. 4 ). As a result, there were significant difference between the control group and febuxostat group in E/e' (treatment $p=0.045$, time, $p=$ 0.077 , treatment $\times$ Time, $p=0.137$; Fig. 4 ) after 24 months (control group 0.15 [0.001-0.291], febuxostat group -0.13
[-0.289-0.031], $p=0.010)$. We confirmed that there is no adverse effect of febuxostat on echocardiographic parameters and the febuxostat may lead to preventing the impaired diastolic dysfunction.

\section{Discussion}

This study was a post hoc subgroup analysis of the PRIZE trial that focused on the effect of febuxostat on echocardiographic variables, including diastolic functional parameters. The key finding of the study was that the addition of febuxostat significantly prevented the worsen 
Table 2 Echocardiographic variables at baseline

\begin{tabular}{lllll}
\hline & ALL & Control & Febuxostat & P value \\
\hline Number & 65 & 34 & 31 & \\
Echocardiographic variables & & & & \\
TMF-E & $66[57,80]$ & $64[57,80]$ & $66[58,81]$ & 0.480 \\
Averaged e' & $7.65[6.25,9.10]$ & $7.75[6.95,10.20]$ & $6.09[4.80,7.99]$ & 0.028 \\
Averaged E/e' & $8.04[7.01,10.01]$ & $7.90[6.32,9.08]$ & $9.18[7.86,11.67]$ & 0.032 \\
TMF-A & $79[67,91]$ & $83[69,93]$ & $74[66,89]$ & 0.430 \\
E/A & $0.79[0.74,1.11]$ & $0.77[0.69,1.13]$ & $0.86[0.76,1.08]$ & 0.302 \\
Deceleration time & $217[192,241]$ & $220[200,247]$ & $210[186,234]$ & 0.349 \\
LV end-diastolic dimension & $48.0[45.0,52.0]$ & $48.2[44.9,52.3]$ & $47.0[45.0,52.0]$ & 0.804 \\
LV end-systolic dimension & $30.1[27.7,34.1]$ & $31.0[27.7,34.0]$ & $30.0[27.8,36.3]$ & 0.946 \\
LV ejection fraction & $63.9[56.4,69.0]$ & $64.0[58.5,69.0]$ & $63.0[56.4,69.0]$ & 0.602 \\
LV mass index & $101[88,123]$ & $102[86,121]$ & $99[91,128]$ & 0.611 \\
Categories of LV hypertrophy & & & & 0.774 \\
Normal & $42.9 \%$ & $47.6 \%$ & $38.1 \%$ & $38.1 \%$ \\
Concentric remodeling & $31.0 \%$ & $23.8 \%$ & $9.5 \%$ & $14.3 \%$ \\
Concentric hypertrophy & $11.9 \%$ & $14.3 \%$ & $54[49,70]$ & 0.923 \\
Eccentric hypertrophy & $14.3 \%$ & $14.3 \%$ & $59[42,77]$ & \\
LA volume & $58[45,77]$ &
\end{tabular}

$T M F-E$ early diastolic transmitral flow wave, $e$ ' early mitral annular velocity, $T M F-A$ late diastolic transmitral flow wave, $L V$ left ventricular, $L A$, left atrial.

Data are presented as median (interquartile range).
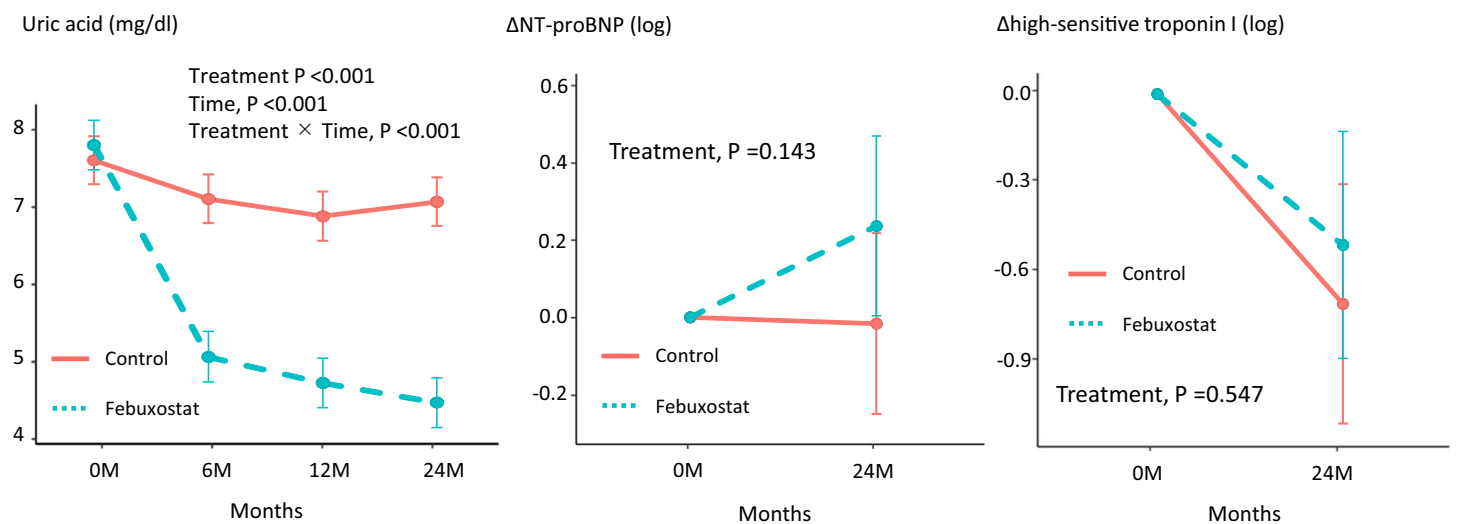

Fig. 2 Changes in uric acid, NT-pro BNP, and high-sensitive troponin I at 12 and 24 months in the two treatment groups

Fig. 3 Changes in left ventricular ejection fraction (LVEF) and LV mass index (LVMI) at 12 and 24 months in the two treatment groups
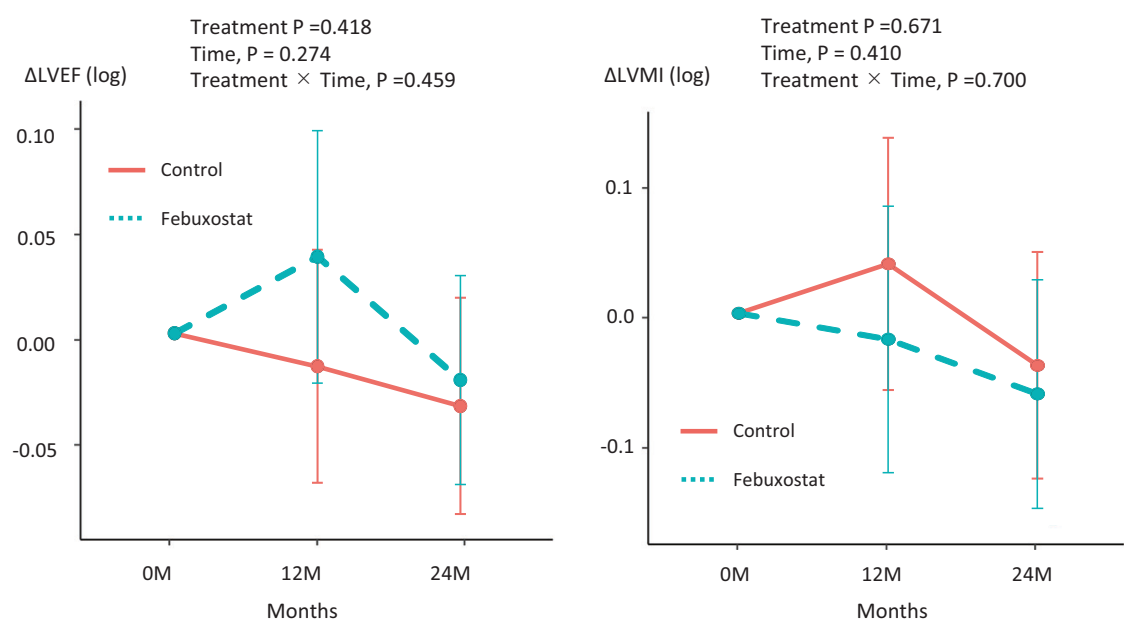

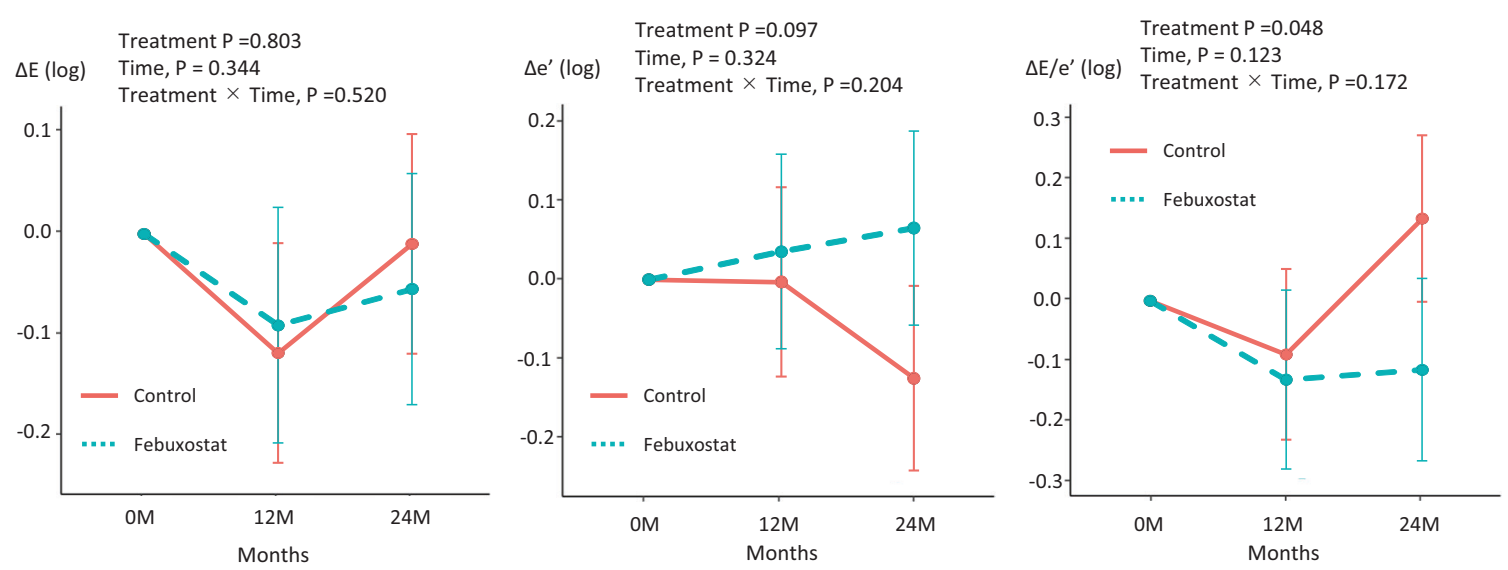

Fig. 4 Changes in early diastolic transmitral flow velocity (E), early diastolic mitral annular velocity (e'), and E/e' at 12 and 24 months in the two treatment groups

diastolic function (e' and $\mathrm{E} / \mathrm{e}^{\prime}$ ) compared to control group (Fig. 5). However, there is no significant differences of other parameters including LV mass index and LVEF between the two groups. Moreover, there is no significant differences in the biomarkers including NT-proBNP and high-sensitive troponin I. According to these results, it seems that febuxostat therapy may have a protective effect on cardiac diastolic function independent from LV morphological changes or biochemical changes. However, the present study should be considered as a proof of concept, and we believe that larger prospective multicenter studies using the advanced diastolic functional markers (e.g., strain imaging) are warranted to confirm the cardiovascular protective effects on diastolic function by febuxostat.

\section{Impact of febuxostat on systolic function}

Elevated SUA has been shown to inhibit nitric oxide production by vascular endothelial cells and also to inhibit their proliferation and migration [15]. In the clinical trial, there are several observational studies and meta-analyses that described elevations of SUA as an independent marker of poor cardiac function, mortality, and poor exercise capacity [16-18]. Increased SUA levels might lead to the echocardiographic abnormalities through impacts on endothelial function and inflammation. Thus, SUA may represent not only an actual prognostic marker of cardiovascular diseases but also a potential target for intervention to treat cardiac function. Several investigators have shown that LV systolic function, such as LVEF and LV mass, improved by treatment of elevated SUA [8, 19]. Our results did not show results consistent with these previous results for the positive effects on LV systolic function. Our data showed that there are no significant differences of LVEF and LV mass between the two groups. One possible explanation was that the baseline LVEF and LV mass were completely within the normal range, then no change occurred during follow-up. We need a prospective randomized study for advanced HF. Another difference between our study and previous studies was that our control subjects underwent only appropriate lifestyle modifications for hyperuricemia.

\section{Impact of febuxostat on diastolic function}

In this study, additional treatment of febuxostat to usual hyperuricemia treatment significantly decreased the annual drop of $\mathrm{e}^{\prime}$ and elevation of E/e', suggesting a preventive effect of febuxostat on LV compliance and diastolic dysfunction. The index of diastolic function is gradually decreased in aging. From the data of reference values, $e^{\prime}$ decreases by $0.1 \mathrm{~cm} / \mathrm{sec}$ per year [20]. In the control group, the $\mathrm{e}^{\prime}$ decreased from 8.5 to 7.7 after 2 years. Thus, there may be a decrease in diastolic function beyond age in patients with asymptomatic hyperuricemia.

Febuxostat treatment did not influence LV systolic functional parameters and cardiac structures or biomarkers, such as NT-proBNP. Some basic studies showed that treatment of SUA decreased myocardial indices of oxidative stress, as well as cardiac tissue xanthine oxidase highlights the potential direct antioxidant effects of the treatment of SUA compound as well as its uric acid and tissue xanthine oxidase lowering properties [21]. Additionally, our study population was consisted of $80 \%$ of all subjects in hypertension. Hyperuricemia is well known to be associated with LV hypertrophy and LV diastolic dysfunction in hypertensive patients $[22,23]$. Hyperuricemia is associated with hypertensive heart disease, and hyperuricemia has an increased risk of coronary or cerebrovascular disease compared to hypertensive patients without hyperuricemia [24]. For these reasons, the protective effects on $\mathrm{LV}$ diastolic dysfunction have been detected in our cohort. However, in our data, the mechanisms for preventive effects of 


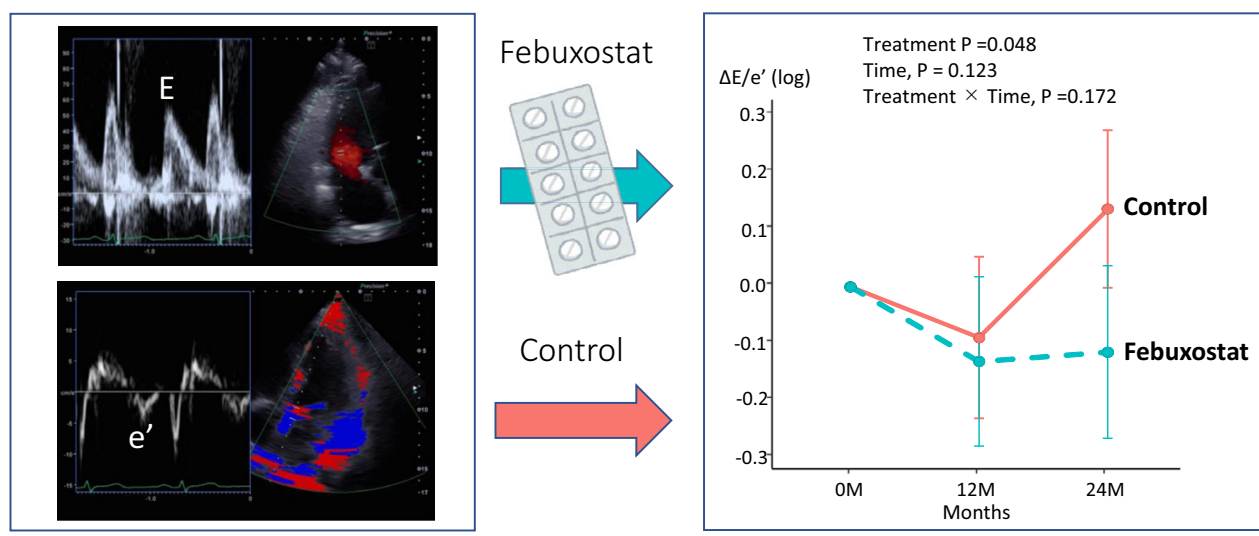

Fig. 5 Graphical Abstract: This study was a post-hoc subgroup analysis of the PRIZE trial that focused on the effect of febuxostat on echocardiographic variables including diastolic functional parameters

febuxostat on diastolic function were not fully explained. This study period may not have been long enough to assess the benefits of febuxostat. Moreover, the treatment effects of febuxostat might be stronger in patients with advanced $\mathrm{HF}$ than in patients with relatively normal cardiac function.

One possible mechanism is that the elevation in serum uric acid could be a reflection of increased xanthine oxidase activity, resulting in abnormal energy metabolism in cardiomyocytes [25]. This pathway might play an important role in diastolic dysfunction. Furthermore, increased activity of xanthine oxidase causes the release of free radicals and increased oxidative stress [26]. Oxidative stress lead to the pathogenesis of diastolic dysfunction. Because the precise mechanisms by which febuxostat treatment suppressed the increase in the diastolic parameter are not confirmed in our study, both basic and clinical data should be assessed in further studies.

Previous studies showed a J-curve association between uric acid and aortic diseases, including hypertension and low levels of SUA might be associated with increased cardiovascular diseases [27, 28]. In our cohort, febuxostat did not adversely affect the LV systolic and diastolic function in patients with asymptomatic hyperuricemia. Thus, in the range of SUA from our cohort, the J-curve association between uric acid and cardiac function was not detected, and lowering medication of hypouricemia should be used in hypouricemia for the cardiac function.

\section{Clinical implication}

In this study, $\mathrm{e}^{\prime}$ decreased and $E / \mathrm{e}^{\prime}$ increased after 24 months in the control group, suggesting LV compliance has been impaired. In contrast, these parameters showed no significant changes in the febuxostat group. Thus, it is suggested that febuxostat at least did not adversely affect the LV compliance; possibly it acted rather protective for the impairment of LV compliance. We were unable to show the strong evidence of febuxostat to the LV diastolic parameters in the present study, it may be accomplished by long-term observation or using advanced HF cohort.

\section{Limitation}

This study was a subanalysis of the PRIZE study and echocardiography was not performed in all subjects. Moreover, the echocardiographic parameters including tricuspid regurgitant jet, pulmonary vein flows, and global longitudinal strains were not performed to assess the diastolic function in detail. Therefore, the number of study subjects was relatively small and included only Japanese patients. Due to the small number of patients, it was statistically difficult to evaluate the relationship between doses and echocardiographic parameters. Further studies enrolling a large number of subjects are needed to confirm the long-term effect of febuxostat on LV diastolic function in patients with hyperuricemia and advanced HF.

\section{Conclusion}

Febuxostat might have a potential of preventable effects on the impaired diastolic dysfunction assessed by echocardiography.

\section{Data availability}

The datasets analyzed during the current study are available from the corresponding authors on reasonable request (contact via prizesub-secre@clin-med.org).

Acknowledgements The authors thank the participants and staff for their contributions to the PRIZE study. The PRIZE study was supported by Teijin Pharma Limited, Japan. The funding body had no role in study design, data collection and analysis, decision to publish or preparation of the manuscript. 
The PRIZE Study Investigators Principal Investigator Koichi Node ${ }^{11}$

PRIZE Steering Committee Toyoaki Murohara ${ }^{12}$, Teruo Inoue $^{13}$, Masataka Sata ${ }^{14}$, Mitsuru Ohishi ${ }^{15}$

PRIZE Executive Committee Kotaro Yokote ${ }^{16}$, Kazuomi Kario ${ }^{17}$, Hirotaka Watada $^{18}$, Iichiro Shimomura ${ }^{19}$, Munehide Matsuhisa ${ }^{14}$, Yoshihiro Fukumoto ${ }^{20}$, Koji Maemura ${ }^{21}$, Yusuke Ohya ${ }^{22}$

PRIZE Site Investigators Yuichi Akasaki ${ }^{15}$, Junya $\mathrm{Ako}^{23}$, Hirohisa Amano $^{13}$, Kazutaka Aonuma ${ }^{24}$, Yutaka Aoyama ${ }^{25}$, Hirofumi Arai ${ }^{26}$, Kuniya Asai $^{27}$, Machiko Asaka ${ }^{11}$, Yoshifumi Awaji ${ }^{28}$, Noriko Ban ${ }^{29}$, Toshiaki $\mathrm{Ban}^{30}$, Yasuko K Bando ${ }^{12}$, Hiroyuki Daida ${ }^{17}$, Shunsuke Eguchi $^{31}$, Mami Enomoto ${ }^{24}$, Yuichi Fujii ${ }^{32}$, Akinori Fujikake ${ }^{33}$, Masanori Fujimoto ${ }^{34}$, Tomohiro Fujisaka ${ }^{35}$, Shuichi Fujita ${ }^{35}$, Satoki Fukae $^{21}$, Daiju Fukuda ${ }^{36}$, Mieko Fukui ${ }^{37}$, Yuhei Goriki ${ }^{38}$, Shuichi Hamasaki $^{39}$, Tomoya Hara ${ }^{36}$, Hiroshi Hasegawa ${ }^{16}$, Kenichi Hashimoto $^{40}$, Mitsumasa Hata ${ }^{41}$, Shiro Hata ${ }^{42}$, Ryo Hayashida ${ }^{12}$, Akihiro Higashi $^{33}$, Seiichiro Higuchi ${ }^{34}$, Akihiro Honda ${ }^{20}$, Satoshi Hoshide ${ }^{17}$, Masaaki Hoshiga ${ }^{35}$, Junko Hotchi ${ }^{36}$, Sachiyo Igata ${ }^{20}$, Yumi Ikehara ${ }^{43}$, Teruo Inoue ${ }^{13}$, Youhei Inoue ${ }^{38}$, Hiroko Ishigami ${ }^{25}$, Masaharu Ishihara $^{44}$, Hideki Ishii ${ }^{12}$, Tetsuya Ishikawa ${ }^{33}$, Takashi Ishimatsu ${ }^{21}$, Yusuke Ishiyama ${ }^{17}$, Takahide Ito $^{35}$, Ayumi Ito $^{25}$, Toshiaki Kadokami $^{45}$, Haruo Kamiya ${ }^{46}$, Soichiro Kashihara ${ }^{45}$, Yoshihiro Kawamura $^{47}$, Kazuo Kitagawa ${ }^{48}$, Yoshio Kobayashi ${ }^{16}$, Satoshi Kodera ${ }^{49}$, Seiji $\operatorname{Koga}^{50}$, Hisashi Koide ${ }^{16}$, Yuji Koide ${ }^{21}$, Hiroshi Koiwaya ${ }^{38}$, Hiroki Kojima $^{12}$, Eri Komai ${ }^{34}$, Takaaki Komatsu ${ }^{33}$, Shingo Kono ${ }^{51}$, Takashi Kono ${ }^{34}$, Yoshiaki Kubota ${ }^{27}$, Akio Kuroda ${ }^{52}$, Takanori Kuroyanagi $^{33}$, Akifumi Kushiyama ${ }^{53}$, Kenya Kusunose ${ }^{36}$, Tatsuya Maruhashi $^{54}$, Kazuo Matsunaga ${ }^{55}$, Tomomi Matsuura ${ }^{36}$, Takafumi Mayama $^{34}$, Daigo Mine ${ }^{56}$, Masatoshi Miyamura ${ }^{35}$, Ryota Morimoto ${ }^{12}$, Hideaki Morita ${ }^{35}$, Hidekazu Nagano ${ }^{16}$. Hidemitsu Nakagawa ${ }^{57}$, Katsunori Nakamura ${ }^{58}$, Ryo Nakamura ${ }^{45}$, Ikuko Nakamura ${ }^{56}$, Hitoshi Nakashima $^{59}$, Mamoru Nanasato ${ }^{31}$, Isao Nishi ${ }^{60}$, Shinichi Niwano ${ }^{23}$, Shuichi Nomura $^{32}$, Nozomu Oda ${ }^{54}$, Shio Oguchi ${ }^{47}$, Mitsutoshi Oguri ${ }^{47}$, Arihide Okahara $^{56}$, Masaaki Okutsu ${ }^{57}$, Fumitake Ozaki ${ }^{33}$, Michishige Ozeki $^{35}$, Tomoko Saisu ${ }^{61}$, Yuichi Saito ${ }^{62}$, Makoto Saitoh ${ }^{63}$, Yosuke $\mathrm{Saka}^{47}$, Yoshihiko Sakai ${ }^{33}$, Kazushi Sakane ${ }^{35}$, Ikki Sakuma ${ }^{34}$, Shakya Sandeep ${ }^{49}$, Hiroaki Sano ${ }^{28}$, Hisakuni Sekino ${ }^{41}$, Yuka Senoo ${ }^{25}$, Kensaku Shibata ${ }^{35}$, Yoshisato Shibata ${ }^{38}$, Takahisa Shibata ${ }^{30}$, Akina Shiga $^{34}$, Kazuki Shiina ${ }^{61}$, Michio Shimabukuro ${ }^{36}$, Yusaku Shimbo ${ }^{12}$, Wataru Shimizu ${ }^{27}$, Masahisa Shimpo ${ }^{17}$, Takeshi Soeki ${ }^{36}$, Koichi Sohmiya $^{35}$, Hiroyuki Suzuki ${ }^{25}$, Susumu Suzuki ${ }^{12}$, Makoto Suzuki ${ }^{64}$, Nobuhiro Tahara ${ }^{20}$, Tazu Tahara ${ }^{53}$, Sadako Takahashi ${ }^{17}$, Bonpei Takase $^{40}$, Kaoru Takegami ${ }^{56}$, Tomoko Takiguchi ${ }^{37}$, Tomonobu Taki$\mathrm{kawa}^{47}$, Ai Tamura ${ }^{34}$, Tomoaki Tanaka ${ }^{16}$, Akihito Tanaka ${ }^{12}$, Hiroyuki Tanaka $^{65}$, Jun Tanigawa ${ }^{35}$, Daisuke Tanimura ${ }^{28}$, Yosuke Tatami ${ }^{12}$, Takashi Terano ${ }^{29}$, Fumio Terasaki ${ }^{35}$, Tomoyuki Tobushi ${ }^{45}$, Seiko Tokoi $^{13}$, Toshiyuki Tsubouchi ${ }^{57}$, Daigaku Uchida ${ }^{66}$, Tomohiro Ueda ${ }^{32}$, Rie Ueno ${ }^{36}$, Hiromi Ueno ${ }^{17}$, Chikara Ueyama ${ }^{67}$, Tetsuzo Wakatsuki ${ }^{36}$, Tomohiko Watanabe ${ }^{35}$, Masato Watarai ${ }^{68}$, Isao Yaguchi ${ }^{33}$, Ayumu Yajima $^{11}$, Jiko Yamada ${ }^{61}$, Kyohei Yamamoto ${ }^{29}$, Sachiko Yamauchi ${ }^{58}$, Yohei Yamauchi ${ }^{35}$, Naoto Yokota ${ }^{69}$, Tomohikov Yoshida ${ }^{29}$, Goro Yoshioka $^{38}$

Members of the Data and Safety Monitoring Board Hiroyuki Daida ${ }^{17}$, Junya $\mathrm{Ako}^{23}$, Kazuo Kitagawa ${ }^{48}$

Members of the Clinical Events Committee Wataru Shimizu ${ }^{27}$, Yoshio Kobayashi $^{16}$, Masaharu Ishihara ${ }^{44}$

Imaging Core Laboratory Tomoko Ishizu ${ }^{70}$

Monitoring Shinichiro Ueda ${ }^{71,72}$
Trial Secretvariat Atsushi Tanaka ${ }^{11}$, Jun-ichi Oyama ${ }^{11}$, Mikiko Kagiyama $^{11,73,74}$

${ }^{11}$ Saga University, Saga, Japan; ${ }^{12}$ Nagoya University Graduate School of Medicine, Nagoya, Japan; ${ }^{13}$ Dokkyo Medical University, Mibu, Japan; ${ }^{14}$ Tokushima University Graduate School, Tokushima, Japan; ${ }^{15}$ Kagoshima University, Kagoshima, Japan; ${ }^{16}$ Chiba University Graduate School of Medicine, Chiba, Japan; ${ }^{17} \mathrm{Jichi}$ Medical University School of Medicine, Shimotsuke, Japan; ${ }^{18}$ Juntendo University Graduate School of Medicine, Tokyo, Japan; ${ }^{19}$ Osaka University, Graduate School of Medicine, Suita, Japan; ${ }^{20}$ Kurume University School of Medicine, Kurume, Japan; ${ }^{21}$ Nagasaki University Graduate School of Bivomedical Sciences, Nagasaki, Japan; ${ }^{22}$ University of the Ryukyus, Okinawa, Japan; ${ }^{23}$ Kitasato University School of Medicine, Sagamihara, Japan; ${ }^{24}$ Graduate School of Comprehensive Human Sciences, University of Tsukuba, Tsukuba, Japan; ${ }^{25}$ Nagoya Daini Red Cross Hospital, Nagoya, Japan; ${ }^{26}$ Kameda Medical Center, Komogawa, Japan; ${ }^{27}$ Nippon Medical School, Tokyo, Japan; ${ }^{28}$ Nagoya Ekisaikai Hospital, Nagoya, Japan; ${ }^{29}$ Chiba Aoba Municipal Hospital, Chiba, Japan; ${ }^{30}$ Isumi Medical Center, Isumi, Japan; ${ }^{31}$ Japanese Red Crossv Nagoya Daini Hospital, Nagoya, Japan; 32Hiroshima General Hospital of West Japan Railway Company, Hiroshima, Japan; ${ }^{33}$ Dokkyo Medical University Saitama Medical Center, Koshigaya, Japan; ${ }^{34}$ Graduate School of Medicine, Chiba University, Chiba, Japan; ${ }^{35}$ Osaka Medical College, Takatsuki, Japan; 36Tokushima University Graduate School of Biomedical Sciences, Tokushima, Japan; ${ }^{37}$ Kimitsu Chuo Hospital, Kisarazu, Japan; 38Miyazaki Medical Association Hospital, Miyazaki, Japan; 39Kagoshima City Hospital, Kagoshima, Japan; ${ }^{40}$ National Defense Medical College, Tokorozawa, Japan; ${ }^{41}$ Sekino Hospital, Tokyo, Japan; ${ }^{42}$ Sasebo City General Hospital, Sasebo, Japan; ${ }^{43}$ University of the Ryuvkyus, Nishihara, Japan; ${ }^{44}$ Hyogo College of Medicine, Nishinomiya, Japan; ${ }^{45}$ Fukuoka Saiseikai Futsukaichi Hospital, Chikushino, Japan; ${ }^{46}$ Japanese Red Cross Nagoya Daiichi Hospital, Nagoya, Japan; ${ }^{47}$ Kasugai Municipal Hospital, Kasugai, Japan; ${ }^{48}$ Tokyo Women's Medical University, Tokyo, Japan; 49Asahi General Hospital, Asahi, Japan; ${ }^{50}$ Nagasaki University Graduate School of Biomedical Sciences, Nagoya, Japan; ${ }^{51}$ Kobe City Medical Center General Hospital, Kobe, Japan; ${ }^{52}$ Institute of Advanced Medical Sciences, Tokushima University, Tokushima, Japan; ${ }^{53}$ The Institute for Adult Diseases, Asahi Life Foundation, Tokyo, Japan; ${ }^{54}$ Graduate School of Biomedical and Health Sciences, Hiroshima University, Hiroshima, Japan; ${ }^{55}$ Imari Arita Kyoritsu Hvospital, Matsuura, Japan; ${ }^{56}$ Saga-Ken Medical Centre Koseikan, Saga, Japan; ${ }^{57}$ Nozaki Tokushukai Hospital, Daito, Japan; ${ }^{58}$ Ryukyu University Hospital, Nishihara, Japan; 59National Hospital Organization Kagoshima Medical Center, Kagoshima, Japan; ${ }^{60}$ National Hospital Organization Kasumigaura Medical Center, Tsuchiura, Japan; ${ }^{61}$ Tokyo Medical University, Tokyo, Japan; ${ }^{62}$ Chiba University Hospital, Chiba, Japan; ${ }^{63}$ Nishio Municipal Hospital, Nishio, Japan; ${ }^{64}$ Kameda Medical Center, Kamogawa, Japan; ${ }^{65}$ Niko Clinic, Takeo, Japan; ${ }^{66}$ Hotaruno Central Clinic, Kisarazu, Japan; ${ }^{67}$ Gifu Prefectural Tajimi vHospital, Tajimi, Japan; ${ }^{68}$ Anjo Kosei Hospital, Anjo, Japan; ${ }^{69}$ Yokota Naika, Miyazaki, Japan; ${ }^{70}$ Tsukuba Echo Core Laboratory. LLC, Tsukuba University, Tsukuba, Japan; ${ }^{71}$ Clinical Research Management Center, University of the Ryukyus, Okinawa, Japan; ${ }^{72}$ Clinical Research Support Center, University of the Ryukyus, Okinawa, Japan; ${ }^{73} \mathrm{Nou}-$ velle Place Inc, Tokyo, Japan; ${ }^{74}$ Organization for Clinical Medicine Promotion, Tokyo, Japan

Author contributions All authors conceptualized this trial. K.K. wrote the draft of the article. H.Y. performed statistical analysis throughout this study. All authors confirmed data collection and study selection 
criteria. All authors enrolled patients and performed study quality assessment. All authors approved the final version of the manuscript.

\section{Compliance with ethical standards}

Conflict of interest A.T. has received honoraria from Boehringer Ingelheim and research funding from GlaxoSmithKline. H.T. received honoraria from Abbott Medical Japan, Bayer, Boehringer Ingelheim, Daiichi Sankyo, Kowa, Ono, Mitsubishi Tanabe and Takeda. Y.F. received research grant from Sanofi KK and Shionogi \& Co. Ltd., honoraria from Public Health Research Foundation, AstraZeneca KK, Eisai Co. Ltd., Kowa Pharmaceutical Co. Ltd., and Ono Pharmaceutical Co. Ltd., and research grant and honoraria from MSD KK, Otsuka Pharmaceutical Co. Ltd., Daiichi Sankyo Co. Ltd., Sumitomo Dainippon Pharma Co. Ltd., Teijin Pharma Ltd., Bayer Yakuhin, Ltd., Mochida Pharmaceutical Co. Ltd., Astellas Pharma Inc., Sanwa Kagaku Kenkyusho Co. Ltd., Takeda Pharmaceutical Co. Ltd., Mitsubishi Tanabe Pharma Corp., and Pfizer Japan Inc. K. Kario. has received research grant, honoraria and consulting fees from Sanwa Kagaku Kenkyusho Co. M.S. has received honoraria from Bayer, Boehringer Ingelheim, Takeda, Astellas, Mochida, Mitsubishi Tanabe, Pfizer, Novartis; research funding from Boehringer Ingelheim; scholarships from Astellas, Takeda, Daiichi Sankyo, MSD, Novartis, Boehringer Ingelheim, AstraZeneca, and Pfizer. K.N. has received research grants from Asahi Kasei, Astellas, Bayer, Boehringer Ingelheim, Mitsubishi Tanabe, Teijin, and Terumo; scholarships from Astellas, Bayer, Bristol-Myers Squibb, Daiichi Sankyo, Daiichi Sankyo Healthcare, Takeda, and Teijin; and personal fees from Astellas, AstraZeneca, Bayer, Boehringer Ingelheim, Daiichi Sankyo Healthcare, Eli Lilly, Kowa, Mitsubishi Tanabe, MSD, Novartis, Ono, Takeda, and Teijin. All other authors declare no competing interests.

No Patient and Public Involvement This research was done without patient involvement. Patients were not invited to comment on the study design and were not consulted to develop patient relevant outcomes or interpret the results. Patients were not invited to contribute to the writing or editing of this document for readability or accuracy.

Publisher's note Springer Nature remains neutral with regard to jurisdictional claims in published maps and institutional affiliations.

Open Access This article is licensed under a Creative Commons Attribution 4.0 International License, which permits use, sharing, adaptation, distribution and reproduction in any medium or format, as long as you give appropriate credit to the original author(s) and the source, provide a link to the Creative Commons license, and indicate if changes were made. The images or other third party material in this article are included in the article's Creative Commons license, unless indicated otherwise in a credit line to the material. If material is not included in the article's Creative Commons license and your intended use is not permitted by statutory regulation or exceeds the permitted use, you will need to obtain permission directly from the copyright holder. To view a copy of this license, visit http://creativecommons. org/licenses/by/4.0/.

\section{References}

1. Feig DI, Kang DH, Johnson RJ. Uric acid and cardiovascular risk. N Engl J Med. 2008;359:1811-21.

2. Feig DI, Mazzali M, Kang DH, Nakagawa T, Price K, Kannelis J, et al. Serum uric acid: a risk factor and a target for treatment? J Am Soc Nephrol. 2006;17(4 Suppl 2):S69-73.
3. Kuwabara M, Niwa K, Hisatome I, Nakagawa T, Roncal-Jimenez CA, Andres-Hernando A, et al. Asymptomatic hyperuricemia without comorbidities predicts cardiometabolic diseases: five-year Japanese cohort study. Hypertension. 2017;69:1036-44.

4. Numa S, Hirai T, Nakagawa K, Ohara K, Fukuda N, Nozawa T, et al. Hyperuricemia and transesophageal echocardiographic thromboembolic risk in patients with atrial fibrillation at clinically low-intermediate risk. Circ J. 2014;78:1600-5.

5. Chiu T-H, Wu P-Y, Huang J-C, Su H-M, Chen S-C, Chang J-M, et al. Hyperuricemia is associated with left ventricular dysfunction and inappropriate left ventricular mass in chronic kidney disease. Diagnostics. 2020;10:514.

6. Pan K-L, Lin J-C, Lin C-L, Chen M-C, Chang S-T, Chung C-M, et al. Impact of gout on left atrial function: a prospective speckletracking echocardiographic study. PLoS one. 2014;9:e108357.

7. Nakanishi K, Daimon M, Yoshida Y, Ishiwata J, Sawada N, Hirokawa M, et al. Serum uric acid level and subclinical left ventricular dysfunction: a community-based cohort study. ESC heart failure. 2020;7:1031-8.

8. Krishnan E, Hariri A, Dabbous O, Pandya BJ. Hyperuricemia and the echocardiographic measures of myocardial dysfunction. Congestive heart failure. 2012;18:138-43.

9. Lin J-C, Lin C-L, Chen M-C, Chang P-J, Chang S-T, Chung C-M, et al. Gout, not hyperuricemia alone, impairs left ventricular diastolic function. Arthritis research \& therapy. 2015;17:1-8.

10. Becker MA, Schumacher HR Jr, Wortmann RL, MacDonald PA, Eustace D, Palo WA, et al. Febuxostat compared with allopurinol in patients with hyperuricemia and gout. New England Journal of Medicine. 2005;353:2450-61.

11. Sezai A, Soma M, Nakata K-I, Hata M, Yoshitake I, Wakui S, et al. Comparison of febuxostat and allopurinol for hyperuricemia in cardiac surgery patients (NU-FLASH Trial). Circulation J. 2013:CJ-13-0082.

12. Tanaka A, Taguchi I, Teragawa H, Ishizaka N, Kanzaki Y, Tomiyama H, et al. Febuxostat does not delay progression of carotid atherosclerosis in patients with asymptomatic hyperuricemia: a randomized, controlled trial. PLoS medicine. 2020;17:e1003095.

13. Oyama J-I, Tanaka A, Sato Y, Tomiyama H, Sata M, Ishizu T, et al. Rationale and design of a multicenter randomized study for evaluating vascular function under uric acid control using the xanthine oxidase inhibitor, febuxostat: the PRIZE study. Cardiovasc Diabetol. 2016;15:1-10.

14. Lang RM, Bierig M, Devereux RB, Flachskampf FA, Foster E, Pellikka PA, et al. Recommendations for chamber quantification: a report from the American Society of Echocardiography's Guidelines and Standards Committee and the Chamber Quantification Writing Group, developed in conjunction with the European Association of Echocardiography, a branch of the European Society of Cardiology. J Am Soc Echocardiogr. 2005;18:1440-63.

15. Kang DH, Park SK, Lee IK, Johnson RJ. Uric acid-induced Creactive protein expression: implication on cell proliferation and nitric oxide production of human vascular cells. J Am Soc Nephrol. 2005;16:3553-62.

16. Hirsch GA, Bottomley PA, Gerstenblith G, Weiss RG. Allopurinol acutely increases adenosine triphospate energy delivery in failing human hearts. J Am College Cardiol. 2012;59:802-8.

17. Tamariz L, Harzand A, Palacio A, Verma S, Jones J, Hare J. Uric acid as a predictor of all-cause mortality in heart failure: a metaanalysis. Congestive Heart Fail. 2011;17:25-30.

18. Wełnicki M, Gorczyca I, Wójcik W, Jelonek O, Maciorowska M, Uziębło-Życzkowska B, et al. Hyperuricemia as a marker of reduced left ventricular ejection fraction in patients with atrial fibrillation: results of the POL-AF registry study. J Clin Med. 2021;10:1829.

19. Nakagomi A, Saiki Y, Noma S, Kohashi K, Morisawa T, Kosugi $\mathrm{M}$, et al. Effects of febuxostat and allopurinol on the inflammation 
and cardiac function in chronic heart failure patients with hyperuricemia. IJC Metabolic Endocrine. 2015;8:46-55.

20. Grue JF, Storve S, Støylen A, Torp H, Haugen BO, Mølmen HE, et al. Normal ranges for automatic measurements of tissue Doppler indices of mitral annular motion by echocardiography. Data from the HUNT3 Study. Echocardiography. 2019;36:1646-55.

21. Jia G, Habibi J, Bostick BP, Ma L, DeMarco VG, Aroor AR, et al. Uric acid promotes left ventricular diastolic dysfunction in mice fed a Western diet. Hypertension. 2015;65:531-9.

22. Milionis HJ, Kakafika AI, Tsouli SG, Athyros VG, Bairaktari ET, Seferiadis KI, et al. Effects of statin treatment on uric acid homeostasis in patients with primary hyperlipidemia. Am Heart J. 2004;148:635-40.

23. Gu J, Fan YQ, Zhang HL, Zhang JF, Wang CQ. Serum uric acid is associated with incidence of heart failure with preserved ejection fraction and cardiovascular events in patients with arterial hypertension. J Clin Hypertens. 2018;20:560-7.

24. Verdecchia P, Schillaci G, Reboldi G, Santeusanio F, Porcellati C, Brunetti P. Relation between serum uric acid and risk of cardiovascular disease in essential hypertension: the PIUMA study. Hypertension. 2000;36:1072-8.

25. Weiss K, Schär M, Panjrath GS, Zhang Y, Sharma K, Bottomley PA, et al. Fatigability, exercise intolerance, and abnormal skeletal muscle energetics in heart failure. Circulation: Heart Fail. 2017;10:e004129.

26. Mátyás C, Németh BT, Oláh A, Török M, Ruppert M, Kellermayer $\mathrm{D}$, et al. Prevention of the development of heart failure with preserved ejection fraction by the phosphodiesterase-5A inhibitor vardenafil in rats with type 2 diabetes. Eur J Heart Fail. 2017;19:326-36.

27. Otaki Y, Watanabe T, Konta T, Watanabe M, Asahi K, Yamagata $\mathrm{K}$, et al. Impact of hyperuricemia on mortality related to aortic diseases: a 3.8-year nationwide community-based cohort study. Sci Rep. 2020;10:1-8.

28. Kawasoe S, Kubozono T, Ojima S, Kawabata T, Miyahara H, Tokushige $\mathrm{K}$, et al. J-shaped curve for the association between serum uric acid levels and the prevalence of blood pressure abnormalities. Hypertens Res. 2021;44:1-8. 